\title{
Spatial Correlation Based Transmit Antenna Selection Assisted Fully Generalized Spatial Modulation for Peer-to-Peer Communications
}

\section{Vinoth Babu Kumaravelu}

Vellore Institute of Technology: VIT University

Gudla Vishnu Vardhan ( $\nabla$ vsquare.tarus@gmail.com )

Aditya Engineering College https://orcid.org/0000-0001-9545-2103

Asha S

Cognizant Technology Solutions India

\section{Prakasam P}

Vellore Institute of Technology: VIT University

Arthi Murugadass

Sreenivasa Institute of Technology and Management Studies

Francisco Rubén Castillo Soria

Autonomous University of San Luis Potosi: Universidad Autonoma de San Luis Potosi

\section{Research Article}

Keywords: Average bit error rate (ABER), Fully generalized spatial modulation (FGSM), Spatial correlation, Spatial modulation (SM), Spectral effciency, Transmit antenna selection (TAS)

Posted Date: July 26th, 2021

DOl: https://doi.org/10.21203/rs.3.rs-698319/v1

License: (c) (1) This work is licensed under a Creative Commons Attribution 4.0 International License. Read Full License 


\title{
Spatial Correlation Based Transmit Antenna Selection Assisted Fully Generalized Spatial Modulation for Peer-to-Peer communications
}

\author{
Vinoth Babu Kumaravelu • Vishnu \\ Vardhan Gudla • Asha S • P Prakasam . \\ Arthi Murugadass - Francisco R. Castillo \\ Soria
}

Received: date / Accepted: date

\begin{abstract}
The next generation technologies like device-to-device (D2D) and small cells employ small scale multiple input multiple output (MIMO) systems for peer-to-peer (P2P) communications. Due to higher spectral and energy efficiencies, spatial modulation (SM) has become one of the dominant next generation technologies. To maximize spectral efficiency and user experience, high rate SM variants like fully generalized spatial modulation (FGSM) can be employed for $\mathrm{P} 2 \mathrm{P}$ applications. Due to insufficient spacing between
\end{abstract}

Vinoth Babu Kumaravelu

Department of Communication Engineering, School of Electronics Engineering

Vellore Institute of Technology, Vellore, India

E-mail: vinothbab@gmail.com

Vishnu Vardhan Gudla

Department of Electronics and Communication Engineering,

Aditya Engineering College (A), Surampalem, Andhra Pradesh, India

E-mail: vsquare.tarus@gmail.com*

Asha S

Programmer Analyst Trainee,

Cognizant Technology Solutions India Pvt. Ltd., Bangalore, India

E-mail: ashasaravanan08@gmail.com

P Prakasam

Department of Communication Engineering, School of Electronics Engineering

Vellore Institute of Technology, Vellore, India

E-mail: prakasam.p@vit.ac.in

Arthi Murugadass

Department of Computer Science and Engineering,

Sreenivasa Institute of Technology and Management Studies, Chittoor, Andhra Pradesh, India

E-mail: arthimdas@gmail.com

Francisco R. Castillo Soria

Telecommunications department, Faculty of Science,

Autonomous University of San Luis Potosi (UASLP) San Luis Potosi, Mexico

E-mail: ruben.soria@uaslp.mx 
antenna elements of devices, access points (AP), millimetre wave (mmWave) and sub- $\mathrm{THz}$ bands of operations, the performance of SM variants are hindered in $\mathrm{P} 2 \mathrm{P}$ scenarios. The average bit error rate (ABER) performance of FGSM is severely degraded by atleast $13 \mathrm{~dB}$ under spatially correlated channel conditions. To enhance the performance of FGSM, three different transmit antenna selection (TAS) schemes are utilized, which eliminate transmit antennas with maximum spatial correlation. First TAS scheme performs antenna selection based on spatial correlation angle alone, whereas other two schemes use channel capacity in addition to spatial correlation angle. Through extensive Monte Carlo simulations, it has been proved that TAS based on spatial correlation (TAS-SC-FGSM) scheme offers a performance gain of at least $8 \mathrm{~dB}$ over conventional FGSM without antenna selection (FGSM-NTAS). TAS-SCFGSM also outperforms other two hybrid TAS schemes at the cost of higher computational complexity.

Keywords Average bit error rate (ABER) - Fully generalized spatial modulation (FGSM) - Spatial correlation - Spatial modulation (SM) · Spectral efficiency · Transmit antenna selection (TAS)

\section{Introduction}

Sixth generation $(6 \mathrm{G})$ networks are expected to support a peak data rate of 1 Tbps and minimize the radio latency up to $0.1 \mathrm{~ms}$ [1]. It should be $10 \mathrm{X}$ times more energy efficient than fifth generation $(5 \mathrm{G})$ networks. It should be extremely ultra-reliable i.e., only one out of one million outage is allowed. Hence, 6G networks are expected to provide exceptional trade-off between spectral efficiency, energy efficiency and latency [2].

Massive MIMO is one of the powerful solutions for $5 \mathrm{G}$ and $6 \mathrm{G}$ [3]. It can support multiple users simultaneously with laser-like beams. Due to less leakage in undesired directions, the interference is drastically reduced. Because of smaller channel variations, the resource allocation is simple. In addition, the array gain enables low power operation and range extension. However, the requirement for more radio frequency ( $\mathrm{RF}$ ) chains decreases the energy efficiency of massive MIMO. Additionally, it also suffers from higher computational complexity, inter channel interference (ICI) and requires inter antenna synchronization (IAS).

$\mathrm{P} 2 \mathrm{P}$ communication enables direct communication between the peers, without routing the information through core network. It is widely used in applications like public safety, emergency communication, Internet of things (IoT), intelligent transportation systems (ITS), proximity based games etc $[4,5]$. It is effective under critical scenarios like no coverage from any of the access networks and damaged wireless infrastructure. Due to resource reuse and hop gains, it is widely used in D2D and small cells [6]. With P2P, peers can directly exchange channel states. The collective information can be fed to base station (BS) or AP. AP can perform precoding to eliminate interference between users under multi-user environment $[7,8]$. The public safety applications 
demand high reliable services with ultra-low latency. Hence, small scale MIMO can be employed for reliable P2P links.

The concept of SM is first introduced by R.Y. Meshleh in 2008 [9]. At any time instant, SM techniques activate either one or few transmit antennas for the transmission of modulated symbol(s). The activated antenna indices (spatial constellation) convey information in addition to signal constellation. Because of lower complexity, higher spectral and energy efficiency, elimination of ICI and no necessity of IAS, SM techniques have gained tremendous attention recently. Hence, SM variants are the best alternative solution for massive MIMO in P2P applications. [10].

The spectral efficiency of SM is proportional to base-two logarithm of the number of transmit antennas. SM yields lower spectral efficiency when operated with massive MIMO i.e., employing huge number of antennas at the transmitter/receiver. In order to improve the spectral efficiency, various SM variants are introduced [11-24]. A comparative study of various SM variants in terms of spectral efficiency, computational complexity, number of active RF chains, advantages and disadvantages is listed in [19].

In [20], permutation index-quadrature spatial modulation (PI-QSM) is proposed, where the permutations of active transmit antennas are used as an additional source of information. The real and imaginary components of two complex modulated symbols are transmitted independently via four active antennas. A rotation angle is introduced between two symbols, which improves the ABER performance. This scheme is proposed for four transmitting antennas and not generalized. The computational complexity is also high.

Four high rate variants of SM like FGSM, fully quadrature spatial modulation (FQSM), fully improved quadrature spatial modulation (FIQSM) and fully improved quadrature space shift keying (FIQSSK) are proposed in [21] and [25], where the number of transmit antennas are varied from one to multiple (maximum all). Hence, the spectral efficiency of these schemes is approximately linear with respect to number of transmit antennas.

The ABER of high rate SM variants need to be maintained below target ABER. According to real-time channel conditions, the modulation modes are adjusted using adaptive modulation (AM) schemes to improve the ABER performance [26-29]. In [30,31], adaptive SM (ASM) schemes are proposed, where suitable modulation schemes are selected for transmission to improve the ABER performance under given bit rate constraint. In [32], another ASM scheme is proposed, which allocates suitable modulation schemes according to given switching thresholds. This scheme is expected to improve ABER performance under a target ABER constraint. As this scheme avoids the transmit antenna index estimation, the achieved ABER goes above target ABER. Moreover, the fixed thresholds limit the spectral efficiency.

Most of the above presented SM and ASM schemes are not designed $\mathrm{P} 2 \mathrm{P}$ applications. Insufficient antenna spacing, especially in compact handheld devices and APs introduces channel correlation, which adversely effects the ABER performance. Hence, impact of spatial correlated channel conditions should be considered for analysis $[18,33]$. In order to meet ultra-high 
data rate, a larger bandwidth is required, which is not available in ultra-high frequency (UHF) band. The next generation networks are expected operate on mmWave and sub- $\mathrm{THz}$ bands. Due to smaller spacing between antennas, the channels corresponding to antennas operating at mmWave and sub- $\mathrm{THz}$ bands are affected by high spatial correlation [34,35].

The correlation limits detection reliability of spatial bits [36]. Due to spatial correlation, the channels between different transmit and receive antennas become almost indistinguishable. Hence in this scenario, ambiguities arises while detection of spatial bits that are used to select active antenna(s), which in turn results in huge increase of ABER. In [35], it is suggested to use generalized spatial modulation (GSM) for massive MIMO, which improves spatial multiplexing gain over SM. But the achievable error performance is degraded due to spatial correlation. In [18] and [22], the performance of SM, GSM are tested under correlated Rayleigh and Rician channel conditions. It is shown that there is a severe degradation in performance, when correlation increases.

The unequal error vulnerability of spatial and signal bits against adverse channel effects has been addressed in a number of prior works [17,37-39]. In [17], Trellis-coded spatial modulation (TCSM), an unequal error protection (UEP) scheme is proposed, which partially compensates the performance degradation under correlated Rayleigh and Rician fading. In [37] and [38], alternative approaches like bit-interleaved coded modulation (BICM) and block Markov superposition coding (BMSC) are proposed, which exhibit higher computational complexity. In [39], Trellis coding based method is proposed, which shows some robustness under these adverse correlated channel conditions. In all these approaches [17,37-39], an encoder-decoder is added to the transceiver chain, which increases latency and computational complexity significantly.

In [40-44], precoding based signal shaping methods are proposed, which provide transmit diversity to improve the performance of SM systems. The designed precoding methods maximize the minimum Euclidean distance of the constellation observed at the receiver to improve the ABER performance. In [45-47], precoding schemes based on amplitude scaling are proposed for space shift keying (SSK). These precoders will work efficiently over time-invariant channels. Also, these schemes fail when partial channel state information (CSI) is available at the transmitter. The performance of these schemes degrade under spatially correlated channels.

In [48], a low cost and low complexity precoding approach is proposed, where different complex scaling coefficients are imposed on transmitted symbols based on the activated antennas. This scheme is tested for time varying Rayleigh and Rician fading channels considering the spatial correlation effects. But there is a marginal increase in the transmitter circuitry compared with coding based approaches [17,37-39].

In [33], an ASM scheme with optimized thresholds is developed and its performance is analysed under spatially correlated channels. To reduce the computational complexity, a simple approximation is included, while deriving the error probability of transmit antenna index estimation. Based on the obtained ABER, the adaptive switching thresholds are optimized with the help 
of Karush Kuhn Tucker (KTT) condition and Newton method. The simulation results prove that this scheme offers superior performance over conventional ASM-MIMO in terms of spectral efficiency and ABER.

These unequal error protection schemes offer limited efficiency in compensating the adverse channel effects, while increasing computational complexity and latency [36]. The precoding approaches are effective for smaller constellations (like binary phase shift keying (BPSK) and quadrature phase shift keying (QPSK)) and smaller number of antennas. Their performance degrade severely for higher order constellations. As precoding is an optimization problem, the complexity increases with the number of transmit antennas and modulation order. Hence, precoding approaches are not practical for high spectral efficiency requirements. Signature spatial modulation (SSM) is proposed in [36], where the transmitted symbols are selected from multiple constellations, each corresponds to one of the transmit antennas. These constellations are derived based on successive geometric interpolation method. These interpolations are optimized in such a way that the inter-constellation minimum Euclidean distance with respect to base constellation is maximized. The inter-constellation distance is also independent of modulation order. The symbols transmitted from different antennas are never same and they are separated with each other at least by minimum inter-constellation Euclidean distance. It has been proved that SSM achieves significant performance gains compared to conventional and precoded SM even under highly correlated channel conditions. But bit-toantenna and bit-to-symbol mapping optimizations are not considered.

In redesigned spatial modulation (ReSM) and modified spatial modulation (MSM), a unique bits-to-antenna index mapping scheme is introduced, which guarantees the transmission of modulated symbols from minimal correlated antennas $[18,22,23]$. These schemes activate a maximum of two transmit antennas. In order to ensure simple and accurate detection, adjacent symbols are transmitted, when two antennas are active. It has been shown that the ABER performance of ReSM is superior to TCSM scheme [17]. A novel bitsto-antenna index mapping scheme called dynamic spatial modulation (DSM) is proposed for $4 \times 4$ and $8 \times 8 \mathrm{MIMO}$ configurations in [19]. In contrast to ReSM and MSM, same modulated symbol is transmitted from the selected two antennas providing diversity gain, thereby improving the ABER performance. This scheme is especially developed for IEEE 802.11 ax standard, which is expected to support $8 \times 8$ MIMO configuration. But the spectral efficiency of ReSM, MSM and DSM are identical to conventional SM. Hence, these schemes are not suitable for high rate $\mathrm{P} 2 \mathrm{P}$ applications.

The concept of conventional ReSM is extended in enhanced ReSM (EReSM) [24], where the phase rotation of transmitted symbols is used to convey an extra information bit. The phase rotation angles are optimized to maximize the minimum Euclidean distance between the constellation points. The phase rotation introduces signal space diversity, which sorts out ambiguities at the receiver while detection. It is shown that EReSM outperforms conventional ReSM by atleast $4 \mathrm{~dB}$. The spectral efficiency of EReSM is only one, more than SM. Hence, not suitable for high rate systems. 
Most of the SM proposals discussed in the literature do not exploit the transmitter antennas to get diversity gains. But through multiple antennas, diversity gain is achieved at the receiver [49,50]. In [35], the performance of SM and SSK are analysed considering spatial correlation into account. In order to exploit transmitter antennas to get diversity gains, a new SM scheme called time-orthogonal signal design assisted SM (TOSD-SM) is proposed. Additionally, to quantify the error performance in the presence of spatial correlation, closed-form expression for ABER is derived.

In [51], an antenna grouped SM (GrSM) scheme is proposed to overcome the shortcomings of SM and GSM schemes under massive MIMO scenarios. Here, transmit antennas are partitioned into different groups. The group with strongly correlated antennas are used to implement SM, whereas the group with weakly correlated antennas are used to obtain multiplexing gain. In order to improve the performance further, AM is combined with GrSM to form adaptive GrSM (AGrSM). This improves the spectral efficiency and error performance. The simulation results prove that GrSM is a promising scheme for massive MIMO, as it outperforms both SM and GSM schemes. The performance of GSM is tested under highly correlated channels such as mmWave and sub-THz bands in [52]. The performance of GSM is severely degraded in correlated channels. In conventional GSM, transmit antennas are selected randomly. In [34], a simplified GSM scheme is proposed for highly correlated channels, where antennas are selected without CSI. An efficient bits-to-index mapping scheme is employed based on gray coding, which reduces spatial ABER. The simulation results show that the proposed scheme outperforms conventional GSM by $1.4 \mathrm{~dB}$ in highly correlated channels. In [53], TAS schemes are employed to full duplex (FD)-SM-MIMO systems. Here, antennas are selected based on channel gains.

In [54], transmit antennas which maximize the minimum Euclidean distance are selected for transmission in SM-MIMO. This scheme is termed as Euclidean distance optimized antenna selection (EDAS). This scheme is optimal, but computational complexity is high due to the requirement of exhaustive search in jointly detecting the antenna subset and modulation order.

In [55], low complexity capacity optimized antenna selection (COAS) is proposed for SM, where the antenna with higher channel amplitude norm is selected for transmission. In [56], EDAS and COAS are tested for massive MIMO. However, these schemes may not show a significant gain over highly correlated channels. The increase in number of users within limited available spectrum, decreases user quality and reliability. Hence to improve the reliability, user selection and TAS schemes are proposed in [57]. The random, capacity and norm based TAS schemes are compared. There is a $17 \%$ improvement in sum rate for TAS schemes over NTAS. In [58], norm and received signal-tonoise ratio (SNR) based TAS schemes are tested for cooperative system with multiple antennas at the source. These schemes offer a $20 \%$ and $37 \%$ improvement over random TAS scheme. But these TAS schemes perform poorly under correlated channels.

In [59], correlation angle between different channel gain vectors are used to 
select transmit antennas for SM. The antennas having higher correlation are discarded. In [60], an antenna selection scheme with the combination of channel amplitude and correlation is proposed for SM. In order to further reduce the complexity, a splitting technique is also introduced, where the computational complexity is drastically reduced compared to the hybrid scheme. All the above optimal and sub-optimal schemes are tested for quadrature spatial modulation (QSM) and double-spatial modulation (D-SM) systems [61,62]. All the correlation based antenna selection schemes are expected to improve the ABER performance of SM variants under highly correlated channels.

The major contributions of the proposed work are as highlighted below:

- The high rate SM variant FGSM coupled correlation based sub-optimal TAS schemes are introduced to attain the spectral and energy efficiency trade-off.

- Two hybrid TAS schemes, which combine channel capacity and spatial correlation angle are proposed to reduce the computational complexity, while maintaining reasonable ABER.

- The proposed schemes are compared by evaluating ABER performance as well as computational complexity.

The remaining sections of the paper is cataloged as follows: System model for FGSM transceiver is briefly explained in Section 2. Section 3 presents various spatial correlation based antenna selection schemes for FGSM system. Computational complexity of all the antenna selection schemes is evaluated in Section 4. In Section 5, Monte Carlo simulation results are presented, where ABER performance of various spatial correlation based antenna selection schemes is evaluated. Finally in Section 6, the paper is concluded.

\subsection{Notations}

Bold and lower case letters denote vectors, whereas bold and upper case letters denote matrices. Lower case letters are used to represent scalars. $\left(\begin{array}{l}. \\ .\end{array}\right)$ denotes binomial coefficient. Norm, Euclidean norm, transpose and Hermitian operations are denoted as $\|\|,.||,.[.]^{T}$ and $(.)^{H}$ respectively.

\section{FGSM transceiver}

The block diagram of FGSM transceiver is indicated in Fig. 1. The incoming block of $\eta$ bits are split into two sub-blocks. The first sub-block of $\left(N_{T x}-1\right)$ bits are used to select active antennas. The second sub-block of $\log _{2} M$ bits are used for modulation mapping. Here, $N_{T x}$ and $M$ are the number of transmitting antennas and modulation order respectively. The generated transmit vector $\mathbf{s}$ is transmitted through the channel. The spectral efficiency of FGSM in bits per channel use (bpcu) is given by [21]

$$
\eta_{\mathrm{FGSM}}=\left(N_{T x}-1\right)+\log _{2}(M)
$$




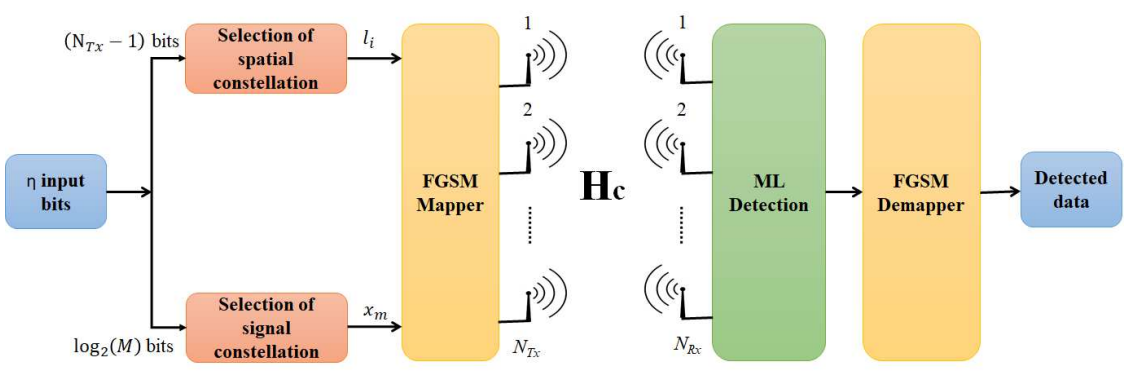

Fig. 1 Block diagram of P2P FGSM transceiver

The second term in Eq.(1) is the information conveyed through signal constellation, whereas the first term is additional information conveyed per channel use through spatial constellation.

In this section, the core idea of FGSM is explained for a sample system with spectral efficiency of 5 bpcu. A sample spatial bit mapping for $N_{T x}=4$ is given in Table 1. As $N_{T x}=4,\left(N_{T x}-1\right)=3$ bits are conveyed through spatial constellation. The mapping of these 3 bits to unique antenna indices is displayed in Table 1. First four, bit combinations are mapped to double antenna subsets and the last four, bit combinations are mapped to single antenna subsets. A sample alternate bit mapping is also shown in Table 2, where all 4 antennas are active for spatial bit combination [000]. The signal constellation mapping for 4-quadrature amplitude modulation (QAM) is shown in Table 3. Since, $M=4, \log _{2}(M)=2$ bits are conveyed through signal constellation.

A transmit vector $\mathbf{s}$ is generated, where selected modulated symbol is loaded at the indices of activated antennas, while a zero is considered at the remaining indices. This transmit vector $\mathbf{s}$ is transmitted through Rayleigh channel $\mathbf{H}$ and distorted by $\mathbf{n}$, which is additive white Gaussian noise (AWGN) with zero mean and variance $\sigma_{n}^{2}$. The received signal vector is given by,

$$
\underbrace{\mathbf{y}}_{N_{R x} \times 1}=\underbrace{\mathbf{H}}_{N_{R x} \times N_{T x}} \underbrace{\mathbf{s}}_{N_{T x} \times 1}+\underbrace{\mathbf{n}}_{N_{R x} \times 1}
$$

Table 1 Spatial constellation mapping for $N_{T x}=4$

\begin{tabular}{ll}
\hline Spatial bits & Antennas selected for transmission \\
\hline 000 & $(1,2)$ \\
001 & $(1,3)$ \\
010 & $(1,4)$ \\
011 & $(2,3)$ \\
100 & 1 \\
101 & 2 \\
110 & 3 \\
111 & 4 \\
\hline
\end{tabular}


Table 2 Alternate spatial constellation mapping for $N_{T x}=4$

\begin{tabular}{ll}
\hline Spatial bits & Antennas selected for transmission \\
\hline 000 & $(1,2,3,4)$ \\
001 & $(1,2,3)$ \\
010 & $(1,2,4)$ \\
011 & $(1,3,4)$ \\
100 & $(2,3,4)$ \\
101 & $(2,3)$ \\
110 & $(2,4)$ \\
111 & $(3,4)$ \\
\hline
\end{tabular}

Table 3 Signal constellation mapping for 4-QAM

\begin{tabular}{lll}
\hline Signal bits & Symbol index & Symbols \\
\hline 00 & $x_{1}$ & $-1+1 j$ \\
01 & $x_{2}$ & $-1-1 j$ \\
10 & $x_{3}$ & $1+1 j$ \\
11 & $x_{4}$ & $1-1 j$ \\
\hline
\end{tabular}

where $\mathbf{H}$ is the channel matrix of size $N_{R x} \times N_{T x}$. Here, $N_{R x}$ is the number of receiver antennas. The elements of $\mathbf{H}$ follow complex Gaussian distribution with zero mean and unit variance. These elements are independent and identically distributed (IID). Under spatially correlated channel conditions, $\mathbf{H}$ in Eq. (2) can be replaced with $\mathbf{H}_{\mathrm{C}}$, which is spatially correlated channel matrix. It can be generated using Kronecker model as [63],

$$
\mathbf{H}_{C}=\mathbf{R}_{R x}^{1 / 2} \mathbf{H R}_{T x}^{1 / 2}
$$

Here, $\mathbf{R}_{T x}$ and $\mathbf{R}_{R x}$ are transmit and receive correlation matrices of size $N_{T x} \times N_{T x}$ and $N_{R x} \times N_{R x}$ respectively. $\mathbf{R}_{T x}$ and $\mathbf{R}_{R x}$ can be obtained using clustered model [64]. The correlation between any two antenna elements $p$ and $q$ (single side, either transmitter or receiver) is calculated using [64],

$$
R_{(p, q)}=\frac{e^{j(D(p-q) \sin (\phi))}}{1+\frac{\theta^{2}}{2}(D(p-q) \cos (\phi))^{2}}, p, q \in\left\{1,2, \ldots, N_{T x} \text { or } N_{R x}\right\}
$$

Here, $D=\frac{2 \pi d}{\lambda}, \lambda$ is the wavelength of operation and $d$ is the separation distance between antenna elements $p$ and $q . \theta$ is the angular spread (AS) and $\phi$ is the angle of arrival (AoA) or angle of departure (AoD).

Consider a bit sequence [10010]. As per Table 1, first 3 bits i.e., [100] select antenna 1 . Next 2 bits i.e., [10] select a symbol $x_{3}=1+1 j$ as per Table 3 . Hence, the transmit vector is generated as $\mathbf{s}=[1+1 j, 0,0,0]^{T}$. This example is for single active antenna. The corresponding received signal vector is given by,

$$
\left[\begin{array}{l}
y_{1} \\
y_{2} \\
y_{3} \\
y_{4}
\end{array}\right]=\left[\begin{array}{llll}
h_{1,1} & h_{1,2} & h_{1,3} & h_{1,4} \\
h_{2,1} & h_{2,2} & h_{2,3} & h_{2,4} \\
h_{3,1} & h_{3,2} & h_{3,3} & h_{3,4} \\
h_{4,1} & h_{4,2} & h_{4,3} & h_{4,4}
\end{array}\right]\left[\begin{array}{c}
x_{3} \\
0 \\
0 \\
0
\end{array}\right]+\left[\begin{array}{c}
n_{1} \\
n_{2} \\
n_{3} \\
n_{4}
\end{array}\right]
$$


The above equation reduces to,

$$
\left[\begin{array}{l}
y_{1} \\
y_{2} \\
y_{3} \\
y_{4}
\end{array}\right]=\left[\begin{array}{l}
h_{1,1} \\
h_{2,1} \\
h_{3,1} \\
h_{4,1}
\end{array}\right] x_{3}+\left[\begin{array}{l}
n_{1} \\
n_{2} \\
n_{3} \\
n_{4}
\end{array}\right]
$$

Consider another bit sequence [01100]. As per Table 1, first 3 bits i.e., [011] select antenna combination $(2,3)$. Next 2 bits i.e., [00] select a symbol $x_{1}=$ $-1+1 j$ as per Table 3 . Hence, the transmit vector is formed as $\mathbf{s}=[0,-1+$ $1 j,-1+1 j, 0]^{T}$. This example is for double active antenna. The corresponding received signal vector is given by,

$$
\left[\begin{array}{l}
y_{1} \\
y_{2} \\
y_{3} \\
y_{4}
\end{array}\right]=\left[\begin{array}{llll}
h_{1,1} & h_{1,2} & h_{1,3} & h_{1,4} \\
h_{2,1} & h_{2,2} & h_{2,3} & h_{2,4} \\
h_{3,1} & h_{3,2} & h_{3,3} & h_{3,4} \\
h_{4,1} & h_{4,2} & h_{4,3} & h_{4,4}
\end{array}\right]\left[\begin{array}{c}
0 \\
x_{1} \\
x_{1} \\
0
\end{array}\right]+\left[\begin{array}{c}
n_{1} \\
n_{2} \\
n_{3} \\
n_{4}
\end{array}\right]
$$

The above equation can be simplified as,

$$
\left[\begin{array}{l}
y_{1} \\
y_{2} \\
y_{3} \\
y_{4}
\end{array}\right]=\left[\begin{array}{l}
h_{1,2} \\
h_{2,2} \\
h_{3,2} \\
h_{4,2}
\end{array}\right] x_{1}+\left[\begin{array}{l}
h_{1,3} \\
h_{2,3} \\
h_{3,3} \\
h_{4,3}
\end{array}\right] x_{1}+\left[\begin{array}{l}
n_{1} \\
n_{2} \\
n_{3} \\
n_{4}
\end{array}\right]
$$

The generalized received vector for $N_{a}$ active antennas is given by,

$$
\mathbf{y}=\left(\sum_{i=1}^{N_{a}} \mathbf{h}_{l_{i}}\right) x_{m}+\mathbf{n}, l_{i} \in\left\{1,2, \ldots, N_{T x}\right\}, m \in\{1,2, \ldots, M\}
$$

Assuming perfect CSI available at the receiver, the antenna indices and symbol index are estimated with the help of maximum likelihood (ML) detector using,

$$
\left[\widehat{a}, \widehat{x}_{m}\right]=\arg \min _{a, x_{m}}\left\|y-\mathbf{h}_{a} x_{m}\right\|^{2}
$$

where $\mathbf{h}_{a}=\left(\sum_{i=1}^{N_{a}} \mathbf{h}_{l_{i}}\right)$ and $a$ is the index of the activated antenna subset.

\section{Spatial correlation based antenna selection schemes for FGSM}

There are various optimal and sub-optimal antenna selection schemes discussed in the literature for conventional MIMO [65-68], SM-MIMO [53-56, 59-62] and massive MIMO [56] systems. They use Euclidean distance, channel capacity and spatial correlation to select antennas at the transmitter or receiver end. The optimal Euclidean distance based schemes offer superior performance with higher computational complexity [54]. Even though, there is an improvement in energy efficiency, they perform poor in terms of latency. It has 
been proved that these optimal and sub-optimal antenna selection schemes improve the energy efficiency of conventional systems by adding antenna selection gains. To improve the performance of FGSM under spatially correlated channels, we have employed FGSM with spatial correlation based antenna selection schemes. In this section, the antenna selection schemes used are elaborated in detail.

\subsection{TAS based on spatial correlation for FGSM (TAS-SC-FGSM)}

The idea of using spatial correlation angle for TAS is first introduced in [59]. Later, the same scheme is tested for QSM in [61]. In the proposed work, this TAS scheme is modified as per FGSM system. The spatial correlation angle based antenna selection is displayed in Algorithm 1.

$N_{\text {Tot }}, N_{T x}, N_{R x}, H_{T o t}, N$ denote total number of transmit antennas, number

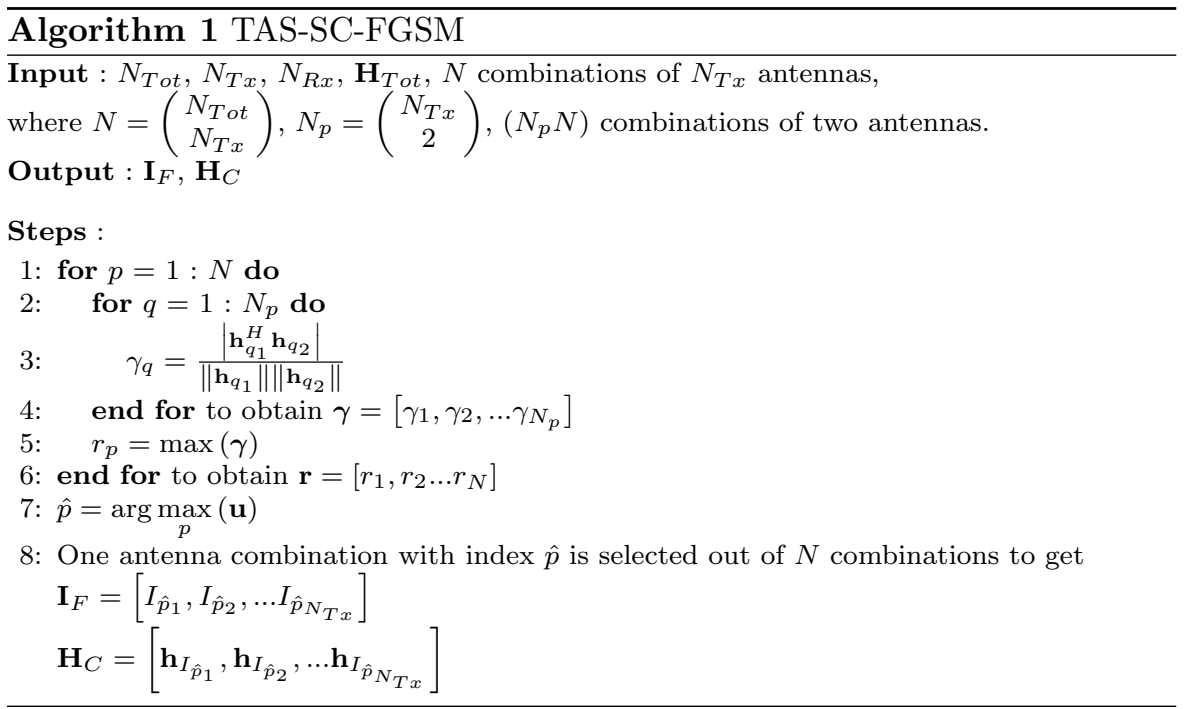

of transmit antennas to be selected, number of receive antennas, IID Rayleigh channel matrix of size $N_{R x} \times N_{T o t}$ and number possible antenna subsets when selecting $N_{T x}$ out of $N_{T o t}$ transmit antennas i.e., $N=\left(\begin{array}{c}N_{T o t} \\ N_{T x}\end{array}\right)$ respectively. The number of possible double antenna combinations for each of the $N$ antenna subsets is given by $N_{p}=\left(\begin{array}{c}N_{T x} \\ 2\end{array}\right)$. Hence, $\left(N_{p} N\right)$ combinations of two antennas are required as the input arguments. The selected antenna subset $\left(\mathbf{I}_{F}\right)$ and the corresponding channel matrix $\left(\mathbf{H}_{C}\right)$ are the outputs. The steps 1 to 6 are enacted for all $N$ combinations of $N_{T x}$ antennas. The steps 2 to 4 are enacted for all $N_{p}$ combinations of double antennas. In step 3, spatial 
correlation angle between two antenna elements $i$ and $j$ are calculated using,

$$
\gamma=\arccos \left(\frac{\left|\mathbf{h}_{i}^{H} \mathbf{h}_{j}\right|}{\left\|\mathbf{h}_{i}\right\|\left\|\mathbf{h}_{j}\right\|}\right)
$$

At the end of step 4, spatial correlation angles for all $N_{p}$ combinations are obtained and stored in $\gamma$. In step 5 , the maximum spatial correlation angle is selected for $p$ th combination of $N_{T x}$ antennas. At the end of step 6, maximum spatial correlation angles for all $N$ combinations are obtained and stored in $\mathbf{r}$. The antenna combination which provides maximum angle of spatial correlation $(\widehat{p})$ is identified in step 7 . The relevant antenna indices $\left(\mathbf{I}_{F}\right)$ and channel matrix $\left(\mathbf{H}_{C}\right)$ are extricated in step 8.

3.2 TAS based on channel capacity and spatial correlation for FGSM (TAS-C-SC-FGSM)

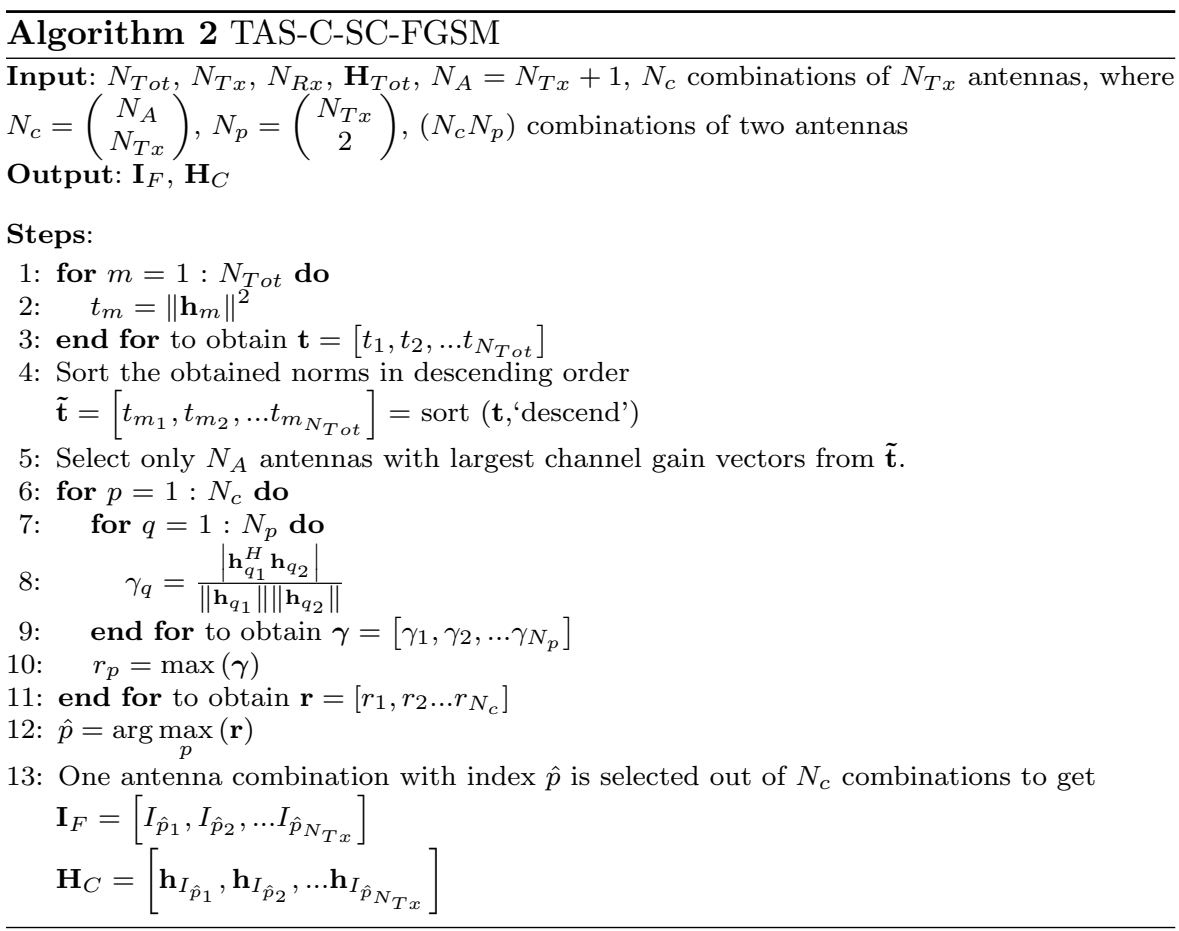

The computational complexity of TAS-SC-FGSM increases with the increase in number of available transmit antennas. In order to reduce the complexity, while maintaining adequate performance, channel capacity is considered in addition to spatial correlation angle for antenna selection. The original algorithm, which is developed for QSM cannot be employed for FGSM. 
Hence, required modifications are carried out and the improvised algorithm is displayed in Algorithm 2.

Steps 1 to 5 are used to select $N_{A}=N_{T x}+1$ antennas from $N_{\text {Tot }}$ based on highest channel capacity norms. In step 2 , norm for each column vector of $H_{T o t}$ is calculated $\left(t_{m}\right)$. Now, $N_{A}$ antennas having largest channel capacity norms are selected out of $N_{\text {Tot }}$ antennas. These $N_{A}$ antennas are considered as the input for spatial correlation based antenna selection, which is executed in steps 6 to 13. This is similar to the TAS-SC-FGSM scheme discussed in Algorithm 1. Earlier $N_{\text {Tot }}$ antennas are the input for TAS-SC-FGSM and now it has been reduced to $N_{A}$. This drastically reduces the computational complexity at the cost of slightly degraded ABER performance.

3.3 TAS based on channel capacity and spatial correlation using splitting mechanism for FGSM (TAS-LC-C-SC-FGSM)

The computational complexity influences the latency performance. To minimize the complexity of TAS-C-SC-FGSM scheme further, a splitting mechanism is introduced, where the channel matrix $\mathbf{H}_{T o t}$ is split into two submatrices $\mathbf{H}_{\text {Tot }}^{1}$ and $\mathbf{H}_{\text {Tot }}^{2}$. Now, TAS-C-SC-FGSM is executed separately for $\mathbf{H}_{T o t}^{1}$ and $\mathbf{H}_{\text {Tot }}^{2}$. Through steps 3 to $7, N_{v}$ antennas with largest channel gain vectors are selected out of $N_{g}$. Then $N_{T x} / 2$ antennas are selected out of $N_{v}$ antennas using spatial correlation angle through steps 8 to 15 . The resultant antenna indices and channel matrices are combined to get $\mathbf{l}_{F}$ and $\mathbf{H}_{C}$. TAS-LC-C-SC-FGSM scheme is shown in Algorithm 3.

\section{Complexity comparison of proposed antenna selection schemes}

The number of real valued multiplications required by these antenna selection schemes are given by

$$
\begin{gathered}
\mathbb{C}_{T A S-S C-F G S M}=\left(\begin{array}{c}
N_{T o t} \\
N_{T x}
\end{array}\right)\left(\begin{array}{c}
N_{T x} \\
2
\end{array}\right)\left(12 N_{R x}+4\right) \\
\mathbb{C}_{T A S-C-S C-F G S M}=4 N_{R x} N_{T o t}+\left(\begin{array}{c}
N_{A} \\
N_{T x}
\end{array}\right)\left(\begin{array}{c}
N_{T x} \\
2
\end{array}\right)\left(12 N_{R x}+4\right) \\
\mathbb{C}_{T A S-L C-C-S C-F G S M}=4 N_{R x} N_{T o t}+2\left(\begin{array}{c}
N_{v} \\
N_{T x} / 2
\end{array}\right)\left(\begin{array}{c}
N_{T x} / 2 \\
2
\end{array}\right)\left(12 N_{R x}+4\right)
\end{gathered}
$$

In Table 4, the computational complexity of different TAS schemes are compared by fixing $N_{T x}=N_{R x}=4, M=4$ and varying $N_{T o t}$. It is clear that the computational complexity of splitting technique is reduced drastically, when compared with TAS-SC-FGSM scheme. 


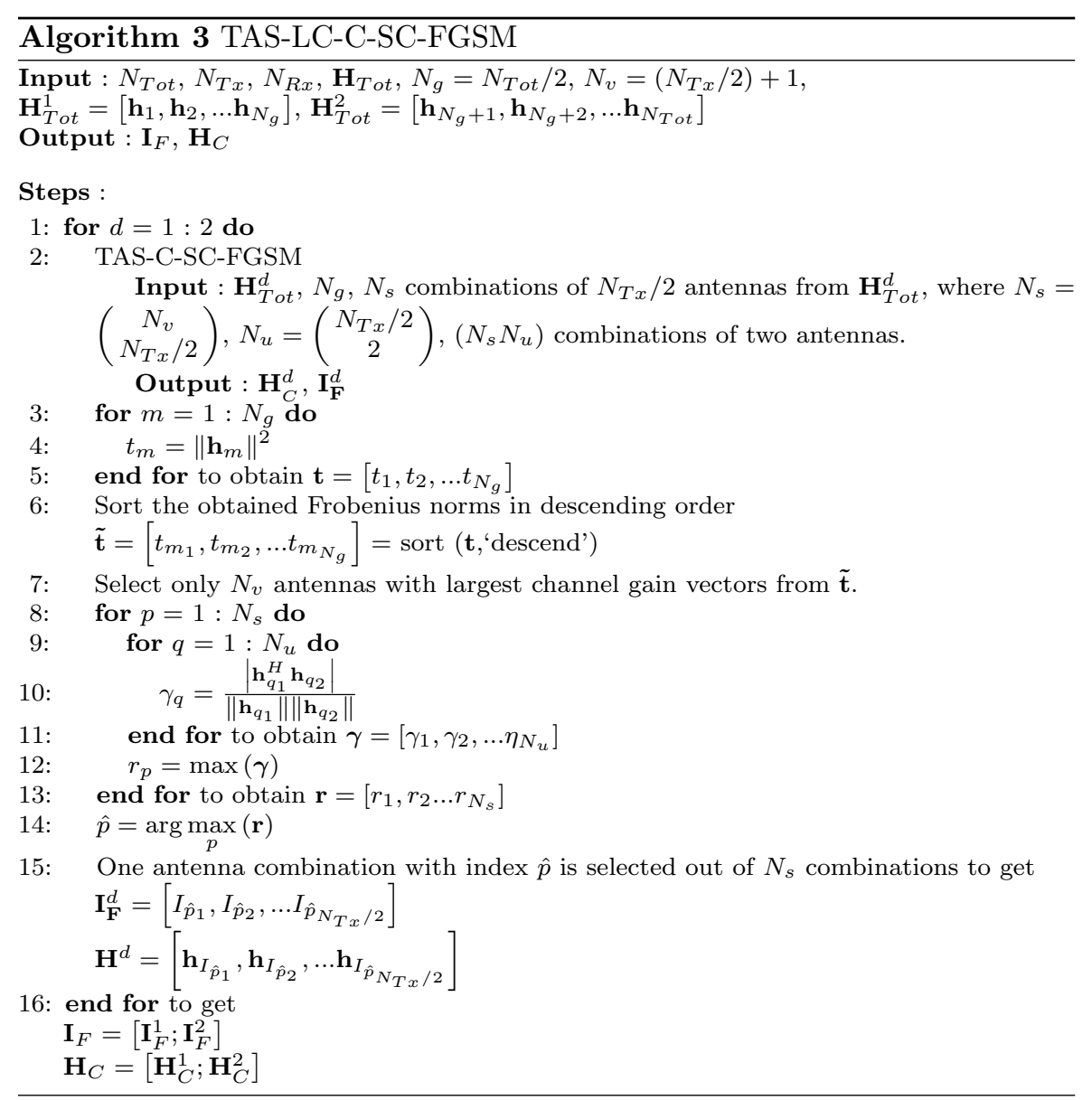

Table 4 Computational complexity of different TAS schemes for varying $N_{T o t}$ and fixed $N_{T x}=4, M=4, N_{R x}=4$

\begin{tabular}{lll}
\hline Scheme & $N_{\text {Tot }}=6$ & $N_{\text {Tot }}=10$ \\
\hline TAS-SC-FGSM & 4680 & 65520 \\
TAS-C-SC-FGSM & 1656 & 1720 \\
TAS-LC-C-SC-FGSM & 408 & 472 \\
\hline
\end{tabular}

\section{Analysis of simulation results}

In this section, the cogency of proposed antenna selection schemes are validated through simulation results. The parameters utilized for simulation study are listed in Table 5. The effect of spatial correlation on the ABER performance of FGSM system is shown in Fig. 2. FGSM under uncorrelated Rayleigh fading channel reaches the target ABER of $10^{-5}$ at the SNR of $\sim 15.5 \mathrm{~dB}$. When the distance between the antenna elements at the transmitter is reduced from $0.5 \lambda$ to $0.1 \lambda$, an additional $\mathrm{SNR}$ of $\sim 13 \mathrm{~dB}$ is required to attain performance 
Table 5 Simulation parameters

\begin{tabular}{ll}
\hline Parameter & Typical value \\
\hline$N_{\text {Tot }}$ & 6,10 \\
$N_{T x}$ & 4 \\
$N_{R x}$ & 4 \\
$\eta$ (bpcu) & 5 \\
$M$ & 4 \\
Modulation scheme & $\mathrm{QAM}$ \\
Number of symbols & $10^{5}$ \\
Fading channel & Uncorrelated Rayleigh, Correlated Rayleigh \\
$d_{T x}$ & $0.1 \lambda$ \\
$d_{R x}$ & $0.1 \lambda, 0.5 \lambda$ \\
AoA & $15^{\circ}$ \\
AoD & $15^{\circ}$ \\
AS & $5^{\circ}$ \\
\hline
\end{tabular}

similar to uncorrelated system. Under highly correlated channel conditions (distance between the antenna elements at both transmitter and receiver is fixed as $0.1 \lambda$ ), an additional SNR of $\sim 18 \mathrm{~dB}$ is required when compared with uncorrelated system. Hence, minimizing the distance between the antenna elements increases the spatial correlation, which in turn degrades the ABER performance of FGSM system significantly.

To combat this loss in SNR, TAS schemes are coupled with FGSM, which yields antenna selection diversity. The performance gains attained by various TAS schemes coupled FGSM are compared with conventional FGSM-NTAS. To simulate Fig. 3, a FGSM system with $N_{T o t}=6, N_{T x}=4, N_{R x}=4$ and $M=4$ are considered. The distance between antenna elements are set to be $d_{T x}=0.1 \lambda$ and $d_{R x}=0.5 \lambda$. All the correlation based TAS schemes perform better than FGSM-NTAS scheme. TAS-SC-FGSM scheme offers superior performance than the hybrid schemes. In hybrid schemes, most of the antenna

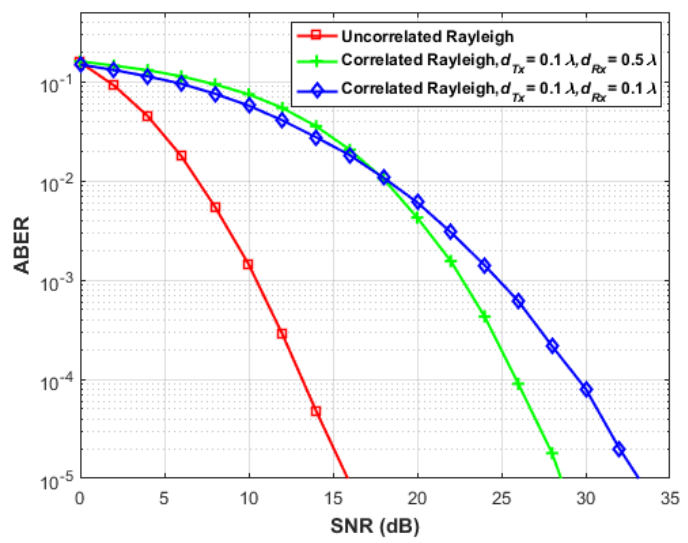

Fig. 2 The effect of spatial correlation on the ABER performance of $4 \times 4$ FGSM system with 4-QAM modulation scheme yielding a spectral efficiency of $5 \mathrm{bpcu}$ 


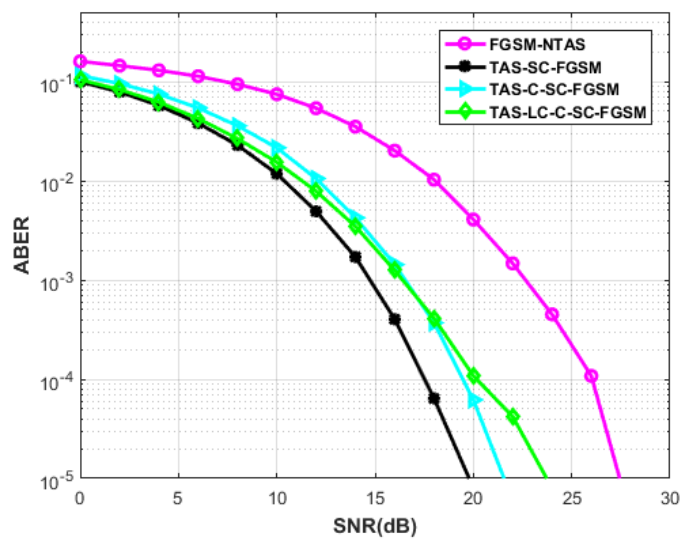

Fig. 3 ABER vs. SNR (dB) comparison between various spatial correlation based TAS schemes for FGSM with configuration $N_{T o t}=6, N_{T x}=4, N_{R x}=4, M=4, d_{T x}=0.1 \lambda$, $d_{R x}=0.5 \lambda$

combinations are eliminated without employing spatial correlation based TAS. Hence, their performance is slightly degraded when compared with TAS-SCFGSM. For low SNR, TAS-C-SC-FGSM and TAS-LC-C-SC-FGSM schemes exhibit similar performance. Whereas under high SNR, there is a slight degradation in the performance of TAS-LC-C-SC-FGSM compared to TAS-C-SCFGSM. This is due to the reduced number of antenna combinations selected through spatial correlation angle. TAS-SC-FGSM scheme achieves SNR gains of $\sim 2 \mathrm{~dB}, \sim 4 \mathrm{~dB}, \sim 8 \mathrm{~dB}$ when compared with TAS-C-SC-FGSM, TAS-LCC-SC-FGSM and FGSM-NTAS respectively.

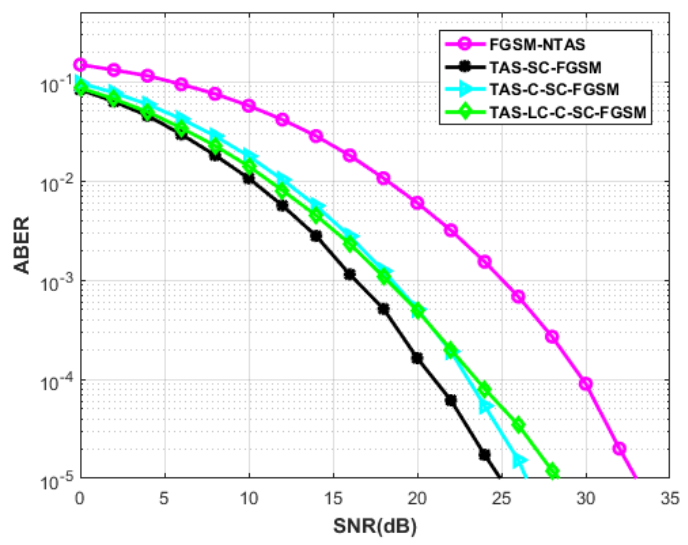

Fig. 4 ABER vs. SNR (dB) comparison between various spatial correlation based TAS schemes for FGSM with configuration $N_{T o t}=6, N_{T x}=4, N_{R x}=4, M=4, d_{T x}=0.1 \lambda$, $d_{R x}=0.1 \lambda$ 


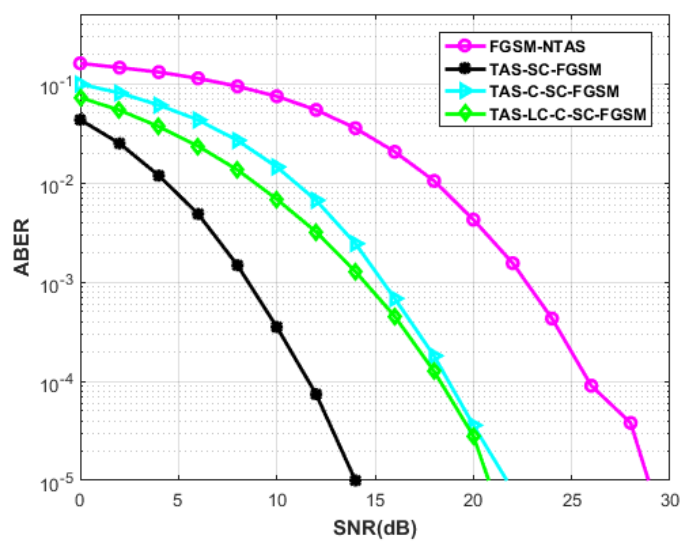

Fig. 5 ABER vs. SNR (dB) comparison between various spatial correlation based TAS schemes for FGSM with configuration $N_{T o t}=10, N_{T x}=4, N_{R x}=4, M=4, d_{T x}=0.1 \lambda$, $d_{R x}=0.5 \lambda$

Similar ABER performance gains are achieved in Fig. 4, where highly correlated channel conditions are considered $\left(d_{T x}=0.1 \lambda, d_{R x}=0.1 \lambda\right)$. TASSC-FGSM scheme achieves SNR gains of $\sim 2 \mathrm{~dB}, \sim 3.5 \mathrm{~dB}, \sim 8.5 \mathrm{~dB}$ when compared with TAS-C-SC-FGSM, TAS-LC-C-SC-FGSM and FGSM-NTAS respectively.

To simulate Fig. 5 all parameters are kept same as used in Fig. 3, except $N_{\text {Tot }}$, which is increased to 10 from 6 . The distance between antenna elements are set to be $d_{T x}=0.1 \lambda$ and $d_{R x}=0.5 \lambda$. The increase in number of available antennas, increase the SNR gains of all the TAS schemes. TAS-SC-

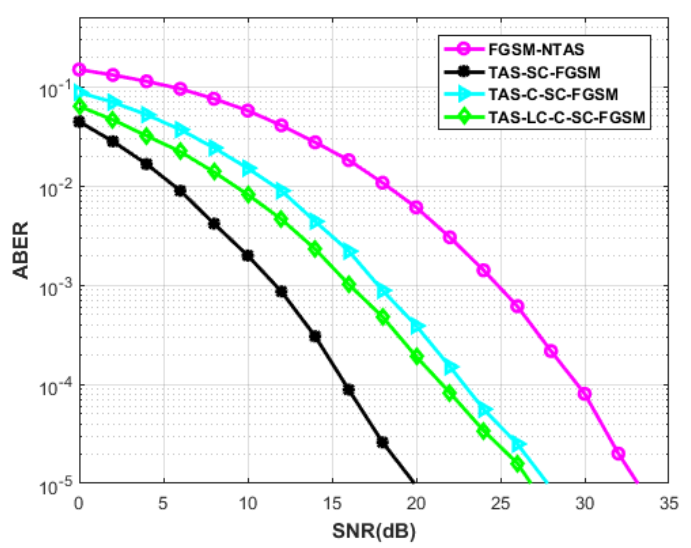

Fig. 6 ABER vs. SNR (dB) comparison between various spatial correlation based TAS schemes for FGSM with configuration $N_{T o t}=10, N_{T x}=4, N_{R x}=4, M=4, d_{T x}=0.1 \lambda$, $d_{R x}=0.1 \lambda$ 
FGSM scheme achieves SNR gains of $\sim 7 \mathrm{~dB}, \sim 7.5 \mathrm{~dB}, \sim 14.5 \mathrm{~dB}$ compared with TAS-C-SC-FGSM, TAS-LC-C-SC-FGSM and FGSM-NTAS respectively. Similar ABER performance gains can be observed in Fig. 6, where highly correlated channel conditions are considered $\left(d_{T x}=0.1 \lambda, d_{R x}=0.1 \lambda\right)$. TASSC-FGSM scheme offers SNR gains of $\sim 8 \mathrm{~dB}, \sim 7 \mathrm{~dB}, \sim 13.5 \mathrm{~dB}$ compared with TAS-C-SC-FGSM, TAS-LC-C-SC-FGSM and FGSM-NTAS respectively. The additional gain in SNR achieved by all the TAS schemes over conventional FGSM-NTAS scheme, for different system configurations is listed in Table 6 .

Table 6 SNR gains achieved by various TAS schemes for a spectral efficiency of $\eta=5 \mathrm{bpcu}$

\begin{tabular}{cllll}
\hline Scheme & \multicolumn{2}{c}{$N_{T o t}=6$} & \multicolumn{2}{c}{$N_{T o t}=10$} \\
\cline { 2 - 5 } & $d_{T x}=0.1 \lambda$, & $d_{T x}=0.1 \lambda$, & $d_{T x}=0.1 \lambda$, & $d_{T x}=0.1 \lambda$, \\
& $d_{R x}=0.5 \lambda$ & $d_{R x}=0.1 \lambda$ & $d_{R x}=0.5 \lambda$ & $d_{R x}=0.1 \lambda$ \\
\hline TAS-SC-FGSM & $\sim 8 \mathrm{~dB}$ & $\sim 8.5 \mathrm{~dB}$ & $\sim 14.5 \mathrm{~dB}$ & $\sim 13.5 \mathrm{~dB}$ \\
TAS-C-SC-FGSM & $\sim 6 \mathrm{~dB}$ & $\sim 7 \mathrm{~dB}$ & $\sim 6 \mathrm{~dB}$ & $\sim 7 \mathrm{~dB}$ \\
TAS-LC-C-SC-FGSM & $\sim 4 \mathrm{~dB}$ & $\sim 5.5 \mathrm{~dB}$ & $\sim 7 \mathrm{~dB}$ & $\sim 7.5 \mathrm{~dB}$ \\
\hline
\end{tabular}

\section{Conclusions}

In this paper, the performances of FGSM coupled correlation based TAS schemes are analysed for dense spatially correlated environments. It is concluded that all the spatial correlation based TAS schemes reduce the SNR requirements to meet the desired ABER. Moreover, all these TAS schemes exhibit lower computational complexity. The spectral efficiency of FGSM is approximately linear with the number of transmit antennas. The proposed spatial correlation based TAS schemes improve the energy efficiency under highly correlated channel conditions. Hence, FGSM coupled spatial correlation based TAS schemes are highly suitable for small scale and massive MIMO based systems. It can be effectively employed for $\mathrm{P} 2 \mathrm{P}$ applications, where reliability is highly important along with spectral and energy efficiencies. The proposed TAS schemes are not tested under indoor line of sight (LoS) Rician fading environment. As a future work, TAS schemes can also be coupled with other high rate SM variants.

\section{Declarations}

Funding: Not Applicable

Conflicts of interest: All the authors certify that they have NO conflicts of interest.

Availability of data and material: Not Applicable

Code availability: Not Applicable

Consent for publication: We, the authors give our consent for the publication of our manuscript in the journal "Wireless Personal Communications". 


\section{References}

1. Matti Latva-aho, Kari Leppänen, Federico Clazzer, and Andrea Munari. Key drivers and research challenges for $6 \mathrm{G}$ ubiquitous wireless intelligence. Technical report, 6G Flagship, University of Oulu, Oulu, 2020.

2. Shangbin Wu Cheng-Xiang Wang Mohammed M. Alwakeel Xiaohu Ge Patcharamaneepakorn, Piya and Marco Di Renzo. Spectral, energy, and economic efficiency of 5G multicell massive mimo systems with generalized spatial modulation. IEEE Transactions on Vehicular Technology, 65(12):9715-9731, 2016.

3. Thomas L Marzetta. Noncooperative cellular wireless with unlimited numbers of base station antennas. IEEE transactions on wireless communications, 9(11):3590-3600, 2010 .

4. Ugljesa Urosevic and Zoran Veljovic. Distributed mimo solutions for peer-to-peer communications in future wireless systems. In 201624 th Telecommunications Forum (TELFOR), pages 1-4. IEEE, 2016.

5. Anil Thomas and Gunasekaran Raja. Finder: A d2d based critical communications framework for disaster management in 5g. Peer-to-Peer Networking and Applications, 12(4):912-923, 2019.

6. Qinghe Du, Meng Liu, Qian Xu, Houbing Song, Li Sun, and Pinyi Ren. Interferenceconstrained routing over p2p-share enabled multi-hop d2d networks. Peer-to-Peer Networking and Applications, 10(6):1354-1370, 2017.

7. Satya Ponnaluri, Sohraab Soltani, Yi Shi, and Yalin Sagduyu. Spectrum efficient communications with multiuser mimo, multiuser detection and interference alignment. In MILCOM 2015-2015 IEEE Military Communications Conference, pages 1473-1478. IEEE, 2015.

8. Malini Balachandran and Noor Mohammed Vali Mohamad. Joint power optimization and scaled beamforming approach in b5g network based massive mimo enabled hetnet with full-duplex small cells. Peer-to-Peer Networking and Applications, 14(1):333-348, 2021.

9. Raed Y Mesleh, Harald Haas, Sinan Sinanovic, Chang Wook Ahn, and Sangboh Yun. Spatial modulation. IEEE Transactions on vehicular technology, 57(4):2228-2241, 2008.

10. Marco Di Renzo, Harald Haas, Ali Ghrayeb, Shinya Sugiura, and Lajos Hanzo. Spatial modulation for generalized mimo: Challenges, opportunities, and implementation. Proceedings of the IEEE, 102(1):56-103, 2013.

11. Abdelhamid Younis, Nikola Serafimovski, Raed Mesleh, and Harald Haas. Generalised spatial modulation. In 2010 conference record of the forty fourth Asilomar conference on signals, systems and computers, pages 1498-1502. IEEE, 2010.

12. Jintao Wang, Shuyun Jia, and Jian Song. Generalised spatial modulation system with multiple active transmit antennas and low complexity detection scheme. IEEE Transactions on Wireless Communications, 11(4):1605-1615, 2012.

13. Abdelhamid Younis. Spatial modulation: Theory to practice. PhD thesis, 2014.

14. Chien-Chun Cheng, Hikmet Sari, Serdar Sezginer, and Yu T Su. Enhanced spatial modulation with multiple constellations. In 2014 IEEE International Black Sea Conference on Communications and Networking (BlackSeaCom), pages 1-5. IEEE, 2014.

15. Raed Mesleh, Salama S Ikki, and Hadi M Aggoune. Quadrature spatial modulation. IEEE Transactions on Vehicular Technology, 64(6):2738-2742, 2014.

16. Raed Mesleh, Omar Hiari, and Abdelhamid Younis. Generalized space modulation techniques: Hardware design and considerations. Physical Communication, 26:87-95, 2018.

17. Raed Mesleh, Marco Di Renzo, Harald Haas, and Peter M Grant. Trellis coded spatial modulation. IEEE Transactions on Wireless Communications, 9(7):2349-2361, 2010.

18. GD Goutham Simha, Shriharsha Koila, N Neha, MANS Raghavendra, and U Sripati. Redesigned spatial modulation for spatially correlated fading channels. Wireless Personal Communications, 97(4):5003-5030, 2017.

19. Gudla Vishnu Vardhan and Kumaravelu Vinoth Babu. Dynamic spatial modulation for next generation networks. Physical Communication, 34:90-104, 2019.

20. Vishnu Vardhan Gudla and Vinoth Babu Kumaravelu. Permutation index-quadrature spatial modulation: A spectral efficient spatial modulation for next generation networks. AEU-International Journal of Electronics and Communications, 111:152917, 2019. 
21. Hany S Hussein, Mohamed Elsayed, Usama Sayed Mohamed, Hamada Esmaiel, and Ehab Mahmoud Mohamed. Spectral efficient spatial modulation techniques. IEEE Access, 7:1454-1469, 2018.

22. Vinoth Babu Kumaravelu, Gaurav Jaiswal, Vishnu Vardhan Gudla, G Ramachandra Reddy, and Arthi Murugadass. Modified spatial modulation: an alternate to spatial multiplexing for 5g-based compact wireless devices. Arabian Journal for Science and Engineering, 44(8):6693-6709, 2019.

23. Gaurav Jaiswal, Vishnu Vardhan Gudla, Vinoth Babu Kumaravelu, G Ramachandra Reddy, and Arthi Murugadass. Modified spatial modulation and low complexity signal vector based minimum mean square error detection for mimo systems under spatially correlated channels. Wireless Personal Communications, 110(2):999-1020, 2020.

24. Vishnu Vardhan Gudla and Vinoth Babu Kumaravelu. Enhanced redesigned spatial modulation: Design and performance evaluation under correlated fading channels. International Journal of Communication Systems, 33(6):e4294, 2020.

25. Yasin Celik. Fully improved quadrature spatial modulation. Arabian Journal for Science and Engineering, pages 1-9, 2021.

26. Mohamed-Slim Alouini and Andrea J Goldsmith. Adaptive modulation over nakagami fading channels. Wireless Personal Communications, 13(1-2):119-143, 2000.

27. Mohammad Torabi. Adaptive modulation for space-frequency block coded ofdm systems. AEU-International Journal of Electronics and Communications, 62(7):521-533, 2008.

28. Xiangbin Yu, Tingting Zhou, Xiaoshuai Liu, Yan Liu, and Dazhuan Xu. Performance of cross-layer design for orthogonal space-time block coded mimo systems with imperfect csi in ricean fading channels. Telecommunication Systems, 57(4):287-294, 2014.

29. Changyou Guo. Research on adaptive modulation of mimo mpsk systems. In 2010 Second International Conference on Networks Security, Wireless Communications and Trusted Computing, volume 1, pages 390-393. IEEE, 2010.

30. Ping Yang, Yue Xiao, Yi Yu, and Shaoqian Li. Adaptive spatial modulation for wireless mimo transmission systems. IEEE Communications Letters, 15(6):602-604, 2011.

31. Ping Yang, Yue Xiao, Yi Yu, Lei Li, Qian Tang, and Shaoqian Li. Simplified adaptive spatial modulation for limited-feedback mimo systems. IEEE transactions on vehicular technology, 62(6):2656-2666, 2013.

32. Zied Bouida, Ali Ghrayeb, and Khalid A Qaraqe. Adaptive spatial modulation for spectrally-efficient mimo systems. In 2014 IEEE Wireless Communications and Networking Conference (WCNC), pages 583-587. IEEE, 2014.

33. Xiangbin Yu, Qing Pan, Yang Li, Yaping Hu, and Tao Liu. Adaptive spatial modulation and thresholds optimization for mimo systems in correlated rayleigh channels. AEUInternational Journal of Electronics and Communications, 89:167-173, 2018.

34. Majed Saad, Feyiz Chris Lteif, Ali Chamas Al Ghouwayel, Hussein Hijazi, Jacques Palicot, and Faouzi Bader. Generalized spatial modulation in highly correlated channels. In 2019 IEEE 30th International Symposium on Personal, Indoor and Mobile Radio Communications (PIMRC Workshops), pages 1-6. IEEE, 2019.

35. Marco Di Renzo and Harald Haas. Performance comparison of different spatial modulation schemes in correlated fading channels. In 2010 IEEE International Conference on Communications, pages 1-6. IEEE, 2010.

36. Mustafa F Özkoç, Mutlu Koca, and Hikmet Sari. Spatial modulation with signature constellations for increased robustness to antenna and channel correlations. Physical Communication, 39:100984, 2020.

37. Mutlu Koca and Hikmet Sari. Bit-interleaved coded spatial modulation. In 2012 IEEE 23rd International Symposium on Personal, Indoor and Mobile Radio Communications-(PIMRC), pages 1949-1954. IEEE, 2012.

38. Zhihua Yang, Chulong Liang, Xiaopei Xu, and Xiao Ma. Block markov superposition transmission with spatial modulation. IEEE Wireless Communications Letters, 3(6):565-568, 2014.

39. Ertugrul Basar, Umit Aygolu, Erdal Panayirci, and H Vincent Poor. New trellis code design for spatial modulation. IEEE transactions on Wireless Communications, 10(8):2670-2680, 2011. 
40. Ping Yang, Yong Liang Guan, Yue Xiao, Marco Di Renzo, Shaoqian Li, and Lajos Hanzo. Transmit precoded spatial modulation: Maximizing the minimum euclidean distance versus minimizing the bit error ratio. IEEE Transactions on Wireless Communications, 15(3):2054-2068, 2015.

41. Ping Yang, Yue Xiao, Bo Zhang, Mohammed El-Hajjar, Shaoqian Li, and Lajos Hanzo. Phase rotation-based precoding for spatial modulation systems. IET Communications, 9(10):1315-1323, 2015.

42. Christos Masouros. Improving the diversity of spatial modulation in miso channels by phase alignment. IEEE Communications letters, 18(5):729-732, 2014.

43. Christos Masouros and Lajos Hanzo. Constellation randomization achieves transmit diversity for single-rf spatial modulation. IEEE Transactions on Vehicular Technology, 65(10):8101-8111, 2015.

44. Ming-Chun Lee, Wei-Ho Chung, and Ta-Sung Lee. Generalized precoder design formulation and iterative algorithm for spatial modulation in mimo systems with csit. IEEE Transactions on Communications, 63(4):1230-1244, 2015.

45. Marco Di Renzo and Harald Haas. Improving the performance of space shift keying (ssk) modulation via opportunistic power allocation. IEEE Communications Letters, 14(6):500-502, 2010.

46. Chien-Hsien Wu, Wei-Ho Chung, and Han-Wen Liang. Improved generalized space-shift keying via power allocation. IEEE communications letters, 18(7):1143-1146, 2014.

47. Adrian Garcia-Rodriguez, Christos Masouros, and Lajos Hanzo. Pre-scaling optimization for space shift keying based on semidefinite relaxation. IEEE Transactions on Communications, 63(11):4231-4243, 2015.

48. Mutlu Koca and Hikmet Sari. Precoding for spatial modulation against correlated fading channels. IEEE Transactions on Wireless Communications, 17(9):5857-5870, 2018.

49. Jeyadeepan Jeganathan, Ali Ghrayeb, and Leszek Szczecinski. Spatial modulation: Optimal detection and performance analysis. IEEE Communications Letters, 12(8):545$547,2008$.

50. Jeyadeepan Jeganathan, Ali Ghrayeb, Leszek Szczecinski, and Andres Ceron. Space shift keying modulation for mimo channels. IEEE Transactions on Wireless Communications, 8(7):3692-3703, 2009.

51. Xingxuan Zuo, Jiankang Zhang, Xiaomin Mu, and Lie-Liang Yang. Channel correlation relied grouped spatial modulation for massive mimo systems. IET Communications, 14(8):1241-1250, 2020.

52. Majed Saad, Faouzi Bader, Jacques Palicot, Ali Chamas Al Ghouwayel, and Hussein Hijazi. Single carrier with index modulation for low power terabit systems. In 2019 IEEE Wireless Communications and Networking Conference (WCNC), pages 1-7. IEEE, 2019.

53. Ba Cao Nguyen, Xuan Nam Tran, et al. Transmit antenna selection for full-duplex spatial modulation multiple-input multiple-output system. IEEE Systems Journal, 2020.

54. Ping Yang, Yue Xiao, Lei Li, Qian Tang, Yi Yu, and Shaoqian Li. Link adaptation for spatial modulation with limited feedback. IEEE Transactions on Vehicular Technology, 61(8):3808-3813, 2012.

55. Rakshith Rajashekar, KVS Hari, and Lajos Hanzo. Antenna selection in spatial modulation systems. IEEE Communications Letters, 17(3):521-524, 2013.

56. Peng Wei, Lu Yin, Yue Xiao, Xu He, and Shaoqian Li. Low-complexity transmit antenna selection in large-scale spatial modulation systems. International Journal of Antennas and Propagation, 2015, 2015.

57. Ashu Taneja and Nitin Saluja. Linear precoding with user and transmit antenna selection. Wireless Personal Communications, 109(3):1631-1644, 2019.

58. A Taneja and N Saluja. A transmit antenna selection based energy-harvesting mimo cooperative communication system. IETE Journal of Research, pages 1-10, 2020.

59. Zhiqiang Zhou, Ning Ge, and Xiaokang Lin. Reduced-complexity antenna selection schemes in spatial modulation. IEEE Communications letters, 18(1):14-17, 2014.

60. Narushan Pillay and HongJun Xu. Low-complexity transmit antenna selection schemes for spatial modulation. IET Communications, 9(2):239-248, 2014. 
61. Suvigya Naidu, Narushan Pillay, and Hongjun Xu. Transmit antenna selection schemes for quadrature spatial modulation. Wireless Personal Communications, 99(1):299-317, 2018.

62. Belal Asaati and Ammar Abu-Hudrouss. Transmit antenna selection schemes for double spatial modulation. TRANSMIT ANTENNA SELECTION SCHEMES FOR DOUBLE SPATIAL MODULATION, 6(06/01), 2020.

63. Claude Oestges. Validity of the kronecker model for mimo correlated channels. In 2006 IEEE 63rd Vehicular Technology Conference, volume 6, pages 2818-2822. IEEE, 2006.

64. François Quitin, Claude Oestges, François Horlin, and Philippe De Doncker. A polarized clustered channel model for indoor multiantenna systems at $3.6 \mathrm{ghz}$. IEEE transactions on vehicular technology, 59(8):3685-3693, 2010.

65. Zhenglan Zhou, Yanjie Dong, Xing Zhang, Wenbo Wang, and Yinghai Zhang. A novel antenna selection scheme in MIMO systems. In 2004 International Conference on Communications, Circuits and Systems (IEEE Cat. No. 04EX914), volume 1, pages 190-194. IEEE, 2004.

66. Yang Yang, Rick S Blum, and Sana Sfar. Antenna selection for mimo systems with closely spaced antennas. EURASIP Journal on Wireless Communications and Networking, 2009(1):739828, 2009.

67. K Vinoth Babu and G Ramachandra Reddy. Quality of service aware inter carrier interference mitigation and antenna selection schemes for beyond $4 \mathrm{G}$ systems. Wireless Personal Communications, 96(1):199-216, 2017.

68. Arthi Murugadass, Arulmozhivarman Pachiyappan, Vinoth Babu Kumaravelu, and Emy Mariam George. Quality of service aware antenna selection scheme for multihop relay networks. AEU-International Journal of Electronics and Communications, $71: 9-20,2017$. 